\title{
Diphtheria Toxoid/Tetanus Toxoid/Acellular Pertussis Vaccine Adsorbed
}

National Cancer Institute

\section{Source}

National Cancer Institute. Diphtheria Toxoid/Tetanus Toxoid/Acellular Pertussis Vaccine Adsorbed. NCI Thesaurus. Code C91717.

A vaccine containing detoxified tetanus toxoid, detoxified diphtheria toxoid and acellular pertussis antigens, adsorbed on aluminum phosphate, with active immunizing activity against diphtheria, tetanus and pertussis. The acellular pertussis vaccine components, produced by Bordetella pertussis, are detoxified pertussis toxin (PT), filamentous hemagg lutinin (FHA), pertactin (PRN) and fimbriae types 2 and 3 (FIM). Intramuscular injection with this vaccine activates the immune system to develop antibodies against tetanus toxin, diphtheria toxin and B. pertussis antigens. 